\title{
MRI-Guided Region-of-Interest Delineation Is Comparable to Manual Delineation in Dopamine Transporter SPECT Quantification in Patients: A Reproducibility Study
}

\author{
Morten Ziebell ${ }^{1}$, Lars H. Pinborg ${ }^{1,2}$, Gerda Thomsen ${ }^{1}$, Robin de Nijs $^{3}$, Claus Svarer ${ }^{1}$, Aase Wagner ${ }^{4}$, and Gitte M. Knudsen ${ }^{1}$ \\ ${ }^{I}$ Neurobiology Research Unit and Cimbi, University of Copenhagen, Rigshospitalet, Copenhagen, Denmark; ${ }^{2}$ Epilepsy Clinic, \\ Department of Neurology, University of Copenhagen, Rigshospitalet, Copenhagen, Denmark; ${ }^{3}$ Department of Clinical Physiology and \\ Nuclear Medicine, University of Copenhagen, Rigshospitalet, Copenhagen, Denmark; and ${ }^{4}$ Department of Diagnostic Radiology, \\ University of Copenhagen, Rigshospitalet, Copenhagen, Denmark
}

\begin{abstract}
A particularly sensitive step in the quantification of SPECT images of the dopamine transporter (DAT) is a correct delineation of the region of interest (ROI). In this study, we primarily compared the reproducibility of the following different approaches for ROI delineation in SPECT images of the DAT: the use of manual delineation (MD) on high-count striatal slides directly on the SPECT image, ROI delineation based on individual MR images (MRD), and oversized striatal ROls - that is, the striatal volume of interest (SVI), as described previously. We also assessed the ability of the different approaches to identify striatal pathology in patients with parkinsonism. Methods: Eight patients with highly variable reductions in cerebral DAT availability were SPECT-scanned twice with 123 I-labeled $N$-(3-iodoprop-(2E)enyl)-2 $\beta$-carboxymethoxy-3 $\beta$-(4'-methylphenyl) nortropane bolus infusion setup and once with an MRI scanner. For SPECT/MRI coregistration, we used external fiducial markers visible on both MRI and SPECT. With the MD and MRD methods, the outcome parameters for DAT availability were the binding potentials and the ratio at equilibrium of specifically bound radioligand to nondisplaceable radioligand in tissue $\left(\mathrm{BP}_{\mathrm{ND}}\right)$. For the SVI method, the outcome parameter was the specific binding ratio (SBR). Results: No statistically significant difference in striatal $\mathrm{BP}_{\mathrm{ND}}$ intraobserver reproducibility was seen among any of the 3 methods. The intraobserver reproducibility average \pm SD for MD was $7.0 \% \pm 4.1 \%$; for MRD, $5.7 \% \pm 5.4 \%$; and for SVI, $6.7 \% \pm 6.0 \%$. Mean intrasubject variability, as determined from the test-retest scans, did not differ with the 3 delineation methods used. The average $( \pm S D)$ intrasubject variability of striatal $\mathrm{BP}_{\mathrm{ND}}$ was $11.9 \% \pm 10.0 \%$ with $\mathrm{MD}$ and $14.6 \% \pm 15.3 \%$ with MRD. With the SVI method, the intrasubject variability of striatal specific binding ratio was $10.0 \% \pm 10.2 \%$. $\mathrm{BP}_{\mathrm{ND}}$ values obtained with the MD and MRD methods were similar (paired $t$ test, $P>0.4$ ). Conclusion: In patients with reduced striatal DAT binding, the reproducibility of the outcome from ROI MD is comparable to both that obtained by delineation of ROI on indi-
\end{abstract}

Received Nov. 12, 2009; revision accepted Mar. 10, 2010.

For correspondence contact: Morten Ziebell, Neurobiology Research Unit, Rigshospitalet, N9201, 9 Blegdamsvej, Copenhagen, DK-2100, Denmark. E-mail: ziebell@nru.dk

COPYRIGHT $\odot 2010$ by the Society of Nuclear Medicine, Inc. vidual MR images, followed by coregistration to the SPECT image, and that obtained with the SVI-based approach.

Key Words: ${ }^{123}$ I-PE2I; dopamine transporters; SPECT; reproducibility; ROI delineation

J Nucl Med Technol 2010; 38:61-68

DOI: 10.2967/jnmt.109.072801

$\mathbf{I}$ maging of cerebral dopamine transporter (DAT) binding is increasingly used as a surrogate marker for the integrity of dopaminergic nerve cells (1-9). Brain imaging of DAT with SPECT constitutes an important adjunct to neurologists' clinical evaluations of patients with movement disorders, particularly in the early stages of the disease (10). For routine clinical purposes, visual inspection and qualitative assessment of the images by an experienced reader may suffice. For research purposes, however, and particularly for longitudinal studies that are designed to evaluate treatment efficacies (11), quantitative estimates of DAT availability are required $(12,13)$.

A particularly important step in DAT quantification is a correct delineation of the region of interest (ROI) in the SPECT image. Overall, there are 3 principally different ways of delineating ROIs on the SPECT image: manual delineation (MD) directly on the SPECT image, templateguided delineation, and delineation based on coregistration with another brain image that provides structural information, such as MRI. In theory, there is only a limited difference between the MD and the adjustable templatebased delineation. Compared with MD, the largest advantage of template-based delineation is that it is less timeconsuming and is operator-independent (14). An alternative to an anatomically correct ROI was described by TossiciBolt et al. in 2006 - the so-called striatal volume-of-interest (SVI) method (15). This method requires no structural 
information on the individual patient's brain but involves a template of oversized ROIs involving all striatal slices. The calculated volume of interest (VOI), the total counts within this volume, the count concentration in a reference region, and a population-based estimate of the average striatal volume are then used to calculate a striatal binding ratio (SBR) equivalent to the ratio of the specific-to-nondisplaceable uptake.

Various studies have attempted to assess different delineation methods by comparing them to a qualitative visual assessment (14). In the absence of a gold standard, however, the most viable way to evaluate the different ROI delineation methods against each other is to perform reproducibility studies. The reproducibility of a method is important not only to the identification of the most suitable method for quantification but also to the appropriate design of studies in terms of, for example, sample size. Reproducibility studies of brain SPECT outcomes of DAT binding have been conducted in both healthy volunteers $(16,17)$ and patients with affected striatal DAT availability (18-21). Intrasubject variability results from both biologic and methodologic variation. In the absence of subjectspecific anatomic information (e.g., cerebral MRI), the ROI delineation is anticipated to be particularly prone to observer bias, but this bias has, to our knowledge, never been formally assessed in SPECT studies of patients with reduced DAT availability.

In healthy volunteers, we showed previously that in terms of the intrasubject variability of the binding potential of DAT, MD of ROIs directly on SPECT images was equally as effective as MRI-defined probability map-based ROI delineation (MRD) (17). The advantage of including MRI-based anatomic information for the assessment of striatal DAT availability in patients with decreased binding is, however, unknown. It is expected that the test-retest variability is greater in patients with reduced DAT availability, because the concentration of tracer in patients' striatal regions is closer to the nonspecific tracer concentrations. In this context, the method for ROI delineation without anatomic information is even more difficult and prone to more observer bias.

The aim of this present study was to compare 3 different methods of ROI delineation on SPECT images of the DAT in patients with reduced DAT availability. The outcome measure was the reproducibility of the delineation (the intraobserver reproducibility) and how large an impact it would have on the overall individual scan-to-scan variability (the intrasubject variability).

\section{MATERIALS AND METHODS}

\section{Patients}

Eight patients with parkinsonism (average age, $69 \mathrm{y}$; age range, 57-80 y; 6 men) were studied twice, at intervals between 14 and $21 \mathrm{~d}$. The patients were included on the day of their assignment for diagnostic ${ }^{123}$ I-labeled $N$-(3-iodoprop-(2E)-enyl)$2 \beta$-carboxymethoxy-3 $\beta$-(4'-methylphenyl) nortropane ( ${ }^{123}$ I-PE2I)
SPECT and asked to enroll in the study if their ${ }^{123}$ I-PE2I SPECT scan was compatible with decreased DAT density. The patients had an average history of 35 mo of neurologic symptoms (range, 12-72 mo). The average scores were 46 (range, 19-72) on the Unified Parkinson Disease Rating Scale, 2.1 (range, 1-3) on the Hoehn and Yahr (H\&Y) scale, and 28.5 (range, 25-30) on the Mini-Mental State Examination. Routine blood test results were normal in all patients. Brain MRIs were unremarkable in all patients. None of the patients was taking antiparkinson drugs before or at the time of the examination. Two patients were taking antidepressant medication (selective serotonin reuptake inhibitors), and their medication was kept unchanged during the test-retest period.

All patients gave informed written consent. The study was performed in accordance with the ethical standards of the Declaration of Helsinki and was approved by the ethical committee of Copenhagen and Frederiksberg (KF 12-009/04).

\section{Experimental Procedures}

As a DAT radiotracer we used ${ }^{123}$ I-PE2I, which is a highly selective DAT ligand with fast kinetics and high target-tobackground ratio (4). Pinborg et al. demonstrated the feasibility of a bolus-plus-constant infusion (B/I) approach for ${ }^{123} \mathrm{I}-\mathrm{PE} 2 \mathrm{I}$ administration (22). The $\mathrm{B} / \mathrm{I}$ approach is superior to a bolus-alone approach because the former allows for the simple and accurate quantification of ${ }^{123}$ I-PE2I binding to DAT. Procedures were performed according to the method of Ziebell et al. (17). Cannulas were inserted into both cubital veins for tracer administration and blood sampling. To block thyroidal uptake of free radioiodine, all patients received $200 \mathrm{mg}$ of potassium perchloride intravenously 30 min before ${ }^{123}$ I-PE2I injection. An average intravenous bolus of $74.3 \mathrm{MBq}$ (range, 65.8-79.9 MBq) of ${ }^{123} \mathrm{I}-\mathrm{PE} 2 \mathrm{I}$ was given, immediately followed by a constant infusion (mean, $96.5 \mathrm{MBq}$; range, $88.6-100.1 \mathrm{MBq}$ ) of ${ }^{123} \mathrm{I}-\mathrm{PE} 2 \mathrm{I}$ for $3 \mathrm{~h}$. The B/I protocol was similar in both studies, with a bolus equal to $2.7 \mathrm{~h}$ (range, 2.6$2.8 \mathrm{~h}$ ) of infusion (the $\mathrm{B} / \mathrm{I}$ ratio).

SPECT was performed with a triple-head IRIX camera (Philips Medical) fitted with low-energy, general-purpose parallel-hole collimators (spatial resolution, $8.5 \mathrm{~mm}$ at $10 \mathrm{~cm}$ ). The mean radius of rotation was $13.9 \mathrm{~cm}$. Each head covered $120^{\circ}$ of the circular orbit. Scans were obtained and stored at fixed angles, with an angular interval of $3^{\circ}$. Six SPECT scans (duration, $10 \mathrm{~min}$ ) were acquired between 120 and $180 \mathrm{~min}$ after injection. The images were reconstructed with a MATLAB 6.5 (The MathWorks)-based program in $128 \times 128$ matrices $(2.33-\mathrm{mm}$ pixels and identical slice thickness) using standard filtered backprojection with a low-pass fourth-order Butterworth filter at 0.3 Nyquist $\left(0.64 \mathrm{~cm}^{-1}\right)$.

High-energy photons of ${ }^{123}$ I penetrated through the lead of the collimator, and Compton scatter in the scintillation crystal caused erroneous counts in the imaging energy window. Therefore, a second energy window positioned at $184-216 \mathrm{keV}$ was used to correct for these downscattered photons in the imaging window (23) (positioned at 143-175 keV). Before reconstruction, the projection images of the second energy window were subtracted from the imaging energy window using a weight of 1.1 (24).

\section{MRI}

All patients underwent 1 structural MRI scan with a $1.5-\mathrm{T}$ Vision scanner (Siemens) using the 3-dimensional magnetizationprepared rapid acquisition gradient-echo sequence (inversion time/ delay time/echo time/repetition time $=300 / 300 / 4.4 / 11.4 \mathrm{~ms}$; flip angle, 12) acquired as sagittal-plane scans with a spatial resolution 
of $1.50 \times 1.13 \times 1.02 \mathrm{~mm}$. There were 130 planes, and the in-plane matrix was $230 \times 256$. The MRI scan and the first SPECT scan were acquired on the same day.

\section{ROIs}

The ROIs were applied to the SPECT images in 3 different ways; the first 2 have already been discussed (17).

$M D$. The ROIs and reference region (cerebellum) were manually delineated directly on the reconstructed SPECT images, guided by the use of Kretschmann and Weinrich's neuroanatomic atlas (25). The direct adaptation of ROI coordinates from the atlas required a reorientation of the reconstructed images to the canthomeatal plane (using, for instance, MATLAB [The MathWorks] or any other program capable of reorienting the reconstructed SPECT images). ROIs were delineated on 5 consecutive slices $(2.3 \mathrm{~mm} / \mathrm{slice})$ in a summed image from all 6 SPECT frames acquired from 120 to $180 \mathrm{~min}$ after injection. All delineations were performed by the same operator with $5 \mathrm{y}$ of experience in ROI delineation on DAT SPECT images.

$M R D$. The ROIs were delineated on the patient's individual MR image and transferred via a coregistration procedure to the individual SPECT image. The actual delineation of the ROIs was computed using a probability map-based method developed by Svarer et al. (26), and cerebellum was used as the reference region. MR and SPECT images were coregistered also using a semiautomatic MATLAB-based method with visual quality inspection, as previously described (17). Corresponding external fiducial markers (5 on each image) were manually identified on the MR and SPECT images. A rigid transformation between the images was then estimated automatically by minimizing the sum of squared errors between the defined points. The MRD software program does not define an overall striatal ROI but delineates only caudate nucleus and putamen as separate ROIs. Because we wanted to compare the reproducibility of striatal specifically bound radioligand to nondisplaceable radioligand in brain tissue $\left(\mathrm{BP}_{\mathrm{ND}}\right)$ between the MRD method and the other ROI-delineating methods, we calculated a volume-weighted striatal ROI for the MRD:

\section{Striatum (MRD)}

$$
=\frac{\left.\left(\text { caudate }[\mathrm{mL}] \times \mathrm{BP}_{\mathrm{ND}, \text { Caudate }}\right)+(\text { putamen }[\mathrm{mL}]) \times \mathrm{BP}_{\mathrm{ND}, \text { Putamen }}\right)}{(\text { caudate }[\mathrm{mL}]+\text { putamen }[\mathrm{mL}])}
$$

For those with special interest, a detailed illustration of the method is available in Svarer et al. (26).
SVI. The original method uses template ROIs large enough to contain all striatal counts, partial volume included, that the operator is required to position (not to delineate) on each side. A 3-dimensional slab (rather than a single slice) ensures the visualization and inclusion of partial-volume counts in the VOIs (15). We adapted this method with the following modifications: instead of constructing a slab-that is, a summed image of all slices with striatal activity and using a template for ROI delineation - we manually delineated the ROIs on the slice with the highest striatal counts and copied these ROIs to all slices with striatal radioactivity. Next, the cerebellum was manually delineated and used as a reference region instead of using the whole brain minus the ROI. And finally, rather than using an average population-based striatal volume (based on postmortem studies) and applying this volume to all individuals, we calculated the striatal volume using each patient's MR image with the MRD method. For those with special interest, a detailed illustration of the method is available in Tossici-Bolt et al. (15).

Figure 1 illustrates the 3 different methods of ROI delineation on SPECT images of the same individual.

\section{Derivation of Binding Parameters and Reproducibility}

The outcome parameter $\mathrm{BP}_{\mathrm{ND}}$ was calculated according to a consensus established by Innis et al. in 2007 (27). At steady state, $\mathrm{BP}_{\mathrm{ND}}=$ specifically bound radioligand/nondisplaceable radioligand in brain tissue.

The SBR was calculated according to the method of TossiciBolt et al. (15):

$$
\mathrm{SBR}=\frac{\mathrm{Ct}_{\mathrm{VOI}} / \mathrm{c}_{\mathrm{r}}-\mathrm{V}_{\mathrm{VOI}}}{\mathrm{V}_{\mathrm{S}}}
$$

where $\mathrm{V}_{\mathrm{S}}$ is the anatomic volume of striatum, $\mathrm{Ct}_{\mathrm{VOI}}$ the total counts in striatal VOI, $c_{r}$ the count concentration in a reference region devoid of receptors or transporters, and $\mathrm{V}_{\mathrm{VOI}}$ the volume of the SVI (which is unitless, like the binding potential).

Because the MRI delineation is semiautomatic, the software provides caudate and putamen regions separately and the striatal binding potential was computed as a volume-weighted average of the caudate nucleus and putamen.

Figure 2 illustrates the study setup. ${ }^{123}$ I-PE2I SPECT scans were delineated for each individual and striatal DAT availability quantified, and the process was repeated $4 \mathrm{wk}$ later. This process was performed on both the first and the second ${ }^{123}$ I-PE2I SPECT

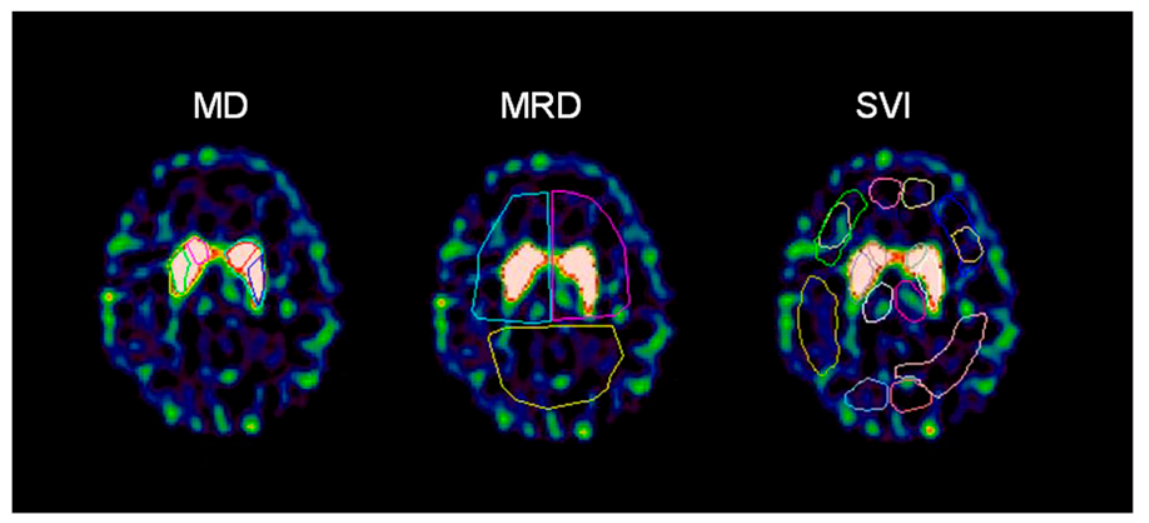

FIGURE 1. Three different methods of ROI delineation on SPECT images of same brain slice in same individual. 


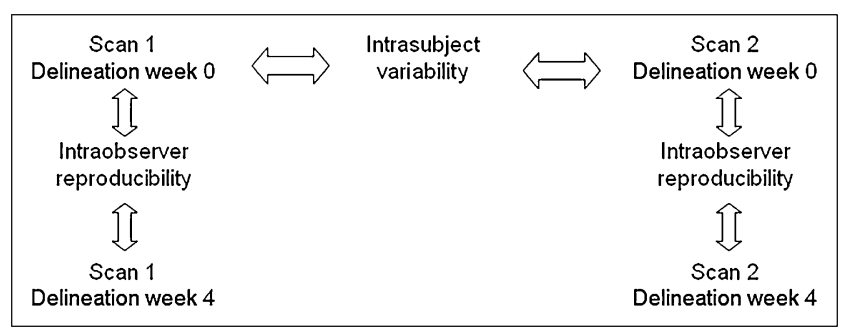

FIGURE 2. Flowchart of study. Eight individuals were scanned twice. Every SPECT $(n=16)$ image was delineated at week 0 , and process was repeated $4 \mathrm{wk}$ later. From these data, intraobserver reproducibility could be calculated according to Equation 2. For every individual, there were 14-21 d between first scan and rescan; from these data $(n=8)$, intrasubject variability was calculated according to Equation 3 .

scan, producing 2 datasets for each region (Fig. 2, left to right, striatum, caudate nucleus, and putamen), 96 total.

The intraobserver reproducibility as a measure of how reproducible the method for ROI delineation is in the same SPECT image was calculated as:

$$
\text { Intraobserver reproducibility }=\frac{\mid \mathrm{BP}(\text { week } 0)-\mathrm{BP}(\text { week } 4) \mid}{(\mathrm{BP}(\text { week } 0)+\mathrm{BP}(\text { week } 4)) / 2} \text {. }
$$

SBR was used instead of binding potential for the SVI method in Equations 2, 3, and 5. As mentioned, both scan 1 and scan 2 were used to calculate the intraobserver reproducibility.

Reproducibility of the ROI volumes within each method was calculated in the same manner as for Equation 2, using the volumes instead of the binding potential.

The intrasubject variability as a measure of the individual ${ }^{123} \mathrm{I}-$ PE2I SPECT scan-to-scan variability was calculated in the same way:

$$
\text { Intrasubject variability }=\frac{\left|\mathrm{BP}_{\mathrm{Scan} 1}-\mathrm{BP}_{\mathrm{Scan} 2}\right|}{\left(\mathrm{BP}_{\mathrm{Scan} 1}+\mathrm{BP}_{\mathrm{Scan} 2}\right) / 2}
$$

where $\mathrm{BP}_{\mathrm{Scan} 1}$ is the binding potential from the first scan (test) and $\mathrm{BP}_{\mathrm{Scan} 2}$ is the binding potential from the second scan (retest).

The reliability of the measures was assessed relative to the between- and within-patient variance by the intraclass correlation coefficient, calculated as in the study by Ichise et al. (28):

$$
\text { Reliability }=\frac{\left(\mathrm{MS}_{\mathrm{B}}-\mathrm{MS}_{\mathrm{W}}\right)}{\left(\mathrm{MS}_{\mathrm{B}}+\mathrm{MS}_{\mathrm{W}}\right)},
$$

where $\mathrm{MS}_{\mathrm{B}}$ is the mean sum of squares between patients and $\mathrm{MS}_{\mathrm{W}}$ the mean sum of squares within patients (17).

The availability of DAT for each patient was also compared with a group of healthy patients. Binding potentials from 25 healthy volunteers, previously scanned with ${ }^{123}$ I-PE2I SPECT, were age-corrected to match the age of the patients, and a $6.6 \%$ reduction per decade was used (29). The age-adjusted binding potential from the 8 patients was then inserted in the following formula:
DAT availability $=$

$$
\frac{\text { Binding potential (patient) }}{\text { Binding potential (age-adjusted normal value) }} \times 100 \% \text {. Eq. } 5
$$

\section{Statistical Analysis}

Linear regression analyses were performed on the intraobserver reproducibility. The slope of linear equations was forced through (0.0). When tested using a Student $t$ test, $P$ values below 0.05 were considered statistically significant. Unless otherwise stated, all values are given as mean $\pm \mathrm{SD}$. Because of the small sample size regarding the intrasubject variability $(n=8)$, we used the nonparametric Wilcoxon matched-pairs signed rank sum test. All statistical analyses were performed with GraphPad Prism (version 5.00v; GraphPad Software Inc.).

\section{RESULTS}

$\mathrm{BP}_{\mathrm{ND}}$ values in the most affected striatal ROI (of the 2 sides), compared with $\mathrm{BP}_{\mathrm{ND}}$ values from our database of 25 age-matched healthy volunteers $(17,22)$, were $8 \%-82 \%$ for MD, 3\%-96\% for MRD, and 26\%-100\% for SVI.

\section{Intraobserver Reproducibility}

The intraobserver reproducibility for each method is shown in Figure 3-that is, the calculated $\mathrm{BP}_{\mathrm{ND}}$ from the first ROI delineation is plotted against the $\mathrm{BP}_{\mathrm{ND}}$ from the second ROI delineation. Table 1 summarizes the intraobserver reproducibility of either $\mathrm{BP}_{\mathrm{ND}}$ or $\mathrm{SBR}$ for all 3 methods; the intraobserver reproducibility was not significantly different among any of the 3 ROI delineation methods (paired $t$ test, $P>0.1$ ).

The intraobserver reproducibility of the mean ROI volumes for MD was $12.3 \% \pm 9.3 \%$ in the striatum, $19.6 \% \pm$ $13.3 \%$ in the caudate nucleus, and $18.3 \% \pm 14.2 \%$ in the putamen. For MRD, the reproducibility was $1.3 \% \pm 1.2 \%$ for the caudate nucleus and $0.9 \% \pm 0.6 \%$ for the putamen.

There was no statistically significant difference (paired $t$ test, $P>0.4)$ in $\mathrm{BP}_{\mathrm{ND}}$ values between the MD and MRD methods (Fig. 4), and a linear regression analysis showed an excellent correlation with a slope of $0.99\left(R^{2}=0.98\right)$. However, the delineated putaminal volumes with the MD method were statistically significantly smaller (4.7 vs. 6.1 $\mathrm{mL})(P<0.001)$ than those delineated with the MRD method. However, the volumes of the caudate nuclei showed no significant difference ( 3.1 vs. $3.0 \mathrm{~mL})(P>0.4)$.

\section{Intrasubject Variability}

Table 2 shows the individual and average striatal intrasubject variability with the 3 different methods, and Table 3 summarizes the sample-based average results for all ROIs. There was no statistically significant difference in the striatal intrasubject variability with the 3 methods (Wilcoxon matched-pairs signed rank sum test, $P>0.2$ ).

We also compared the 3 ROI delineation methods in terms of reproducibility for the single individual-that is, whether all 3 methods provided a consistent picture of a patient's intrasubject variability. A statistically significant 

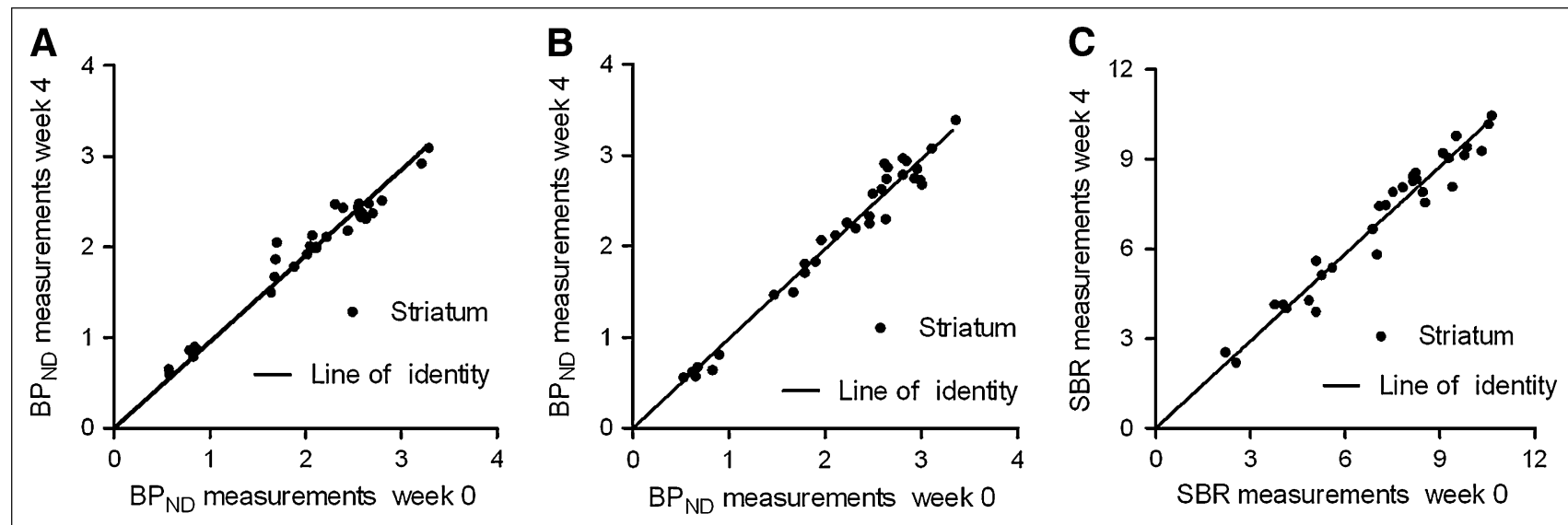

FIGURE 3. Intraobserver reproducibility of $\mathrm{BP}_{\mathrm{ND}}$ in striatum for different delineation methods. MD (A), probability map-based delineation (B), and SVI (C). For all 3 methods, both test and retest scans were quantified (32 data points).

correlation was found only between the MRD and the SVI methods (Wilcoxon matched-pairs signed rank sum test, $P<0.01)$.

To conduct an absolute quantification using the SVI method, the exact size of the individual striatum volume is needed. We wanted to investigate the actual difference in striatal volume in this small sample of patients. The actual average striatal size, computed using the individual MRI scans, was $9.0 \mathrm{~mL}$ (range, $7.2-10.4 \mathrm{~mL}$ ).

\section{DISCUSSION}

The aim of the present study was to compare 3 different types of ROI application methods on SPECT images of the DAT. Outcome measures were reproducibility of the delineation and how reproducibility might affect scan-to-scan variability in patients with reduced DAT density. Our patients, compared with the healthy population, had a DAT availability reduced between $5 \%$ and $85 \%$.

The intraobserver reproducibility ranged from $5.7 \%$ to $7 \%$, and there was no significant difference among any of the methods. The number of patients in this study is low, however, with 2 scannings per subject the number of ROIs reaches 16 . The performance of the intraobserver reproducibility is considerable; thus, for all 3 methods approx- imately 50\% contribution of the overall intrasubject variability in patients with decreased DAT availability stems from the intraobserver reproducibility. The MD method is obviously operator-dependent and dependent on experience. We did not attempt to address the interobserver reproducibility in this study, but assessment of interobserver reproducibility in the individual SPECT centers is recommended if MD is the chosen method.

In theory, the SVI approach and other template methods facilitate the delineation of ROIs, are automated and thereby independent of experienced readers, and are more objective and less sensitive to partial-volume effects than MD. Our results show that the SVI method did not result in a significantly better intraobserver reproducibility than the 2 other methods. We implemented modifications using a different reference region and the individually determined striatal volumes (based on MRIs) and using ROI MD instead of a template. As can be seen from Equation 1, exchanging a population-based average striatal volume with the MRI-determined individual striatal volume did not affect the reproducibility. By contrast, the absolute value of SBR is linearly related to the actual volume and we found a quite high interindividual variation in the MRIdetermined striatal volumes, ranging from 7.2 to $10.4 \mathrm{~mL}$, suggesting that the outcome parameter SBR is determined

TABLE 1

Summed Intraobserver Reproducibility of 3 Different Methods

\begin{tabular}{llccc}
\hline Method & Caudate nucleus & Putamen & Striatum \\
\hline MD (BP ND) $_{\text {MRD }}\left(\mathrm{BP}_{\mathrm{ND}}\right)$ & $10.2 \% \pm 9.2 \%$ & $9.7 \% \pm 5.4 \%$ & $7.0 \% \pm 4.1 \%$ & 0.97 \\
SVI (SBR) & $14.2 \% \pm 12.3 \%$ & $8.1 \% \pm 7.5 \%$ & $5.7 \% \pm 5.4 \%^{*}$ & 0.98 \\
& & & $6.7 \% \pm 6.0 \%$
\end{tabular}

${ }^{*}$ Calculated striatum $=$ volume-weighted (caudate nucleus + putamen).

ICC = intraclass correlation coefficient.

No statistically significant better intraobserver reproducibility was observed for any method (MD vs. MRD, MD vs. SVI, MRD vs. SVI; $P>0.1)$, and all performed equally for intraclass correlation coefficient $(n=16)$. 


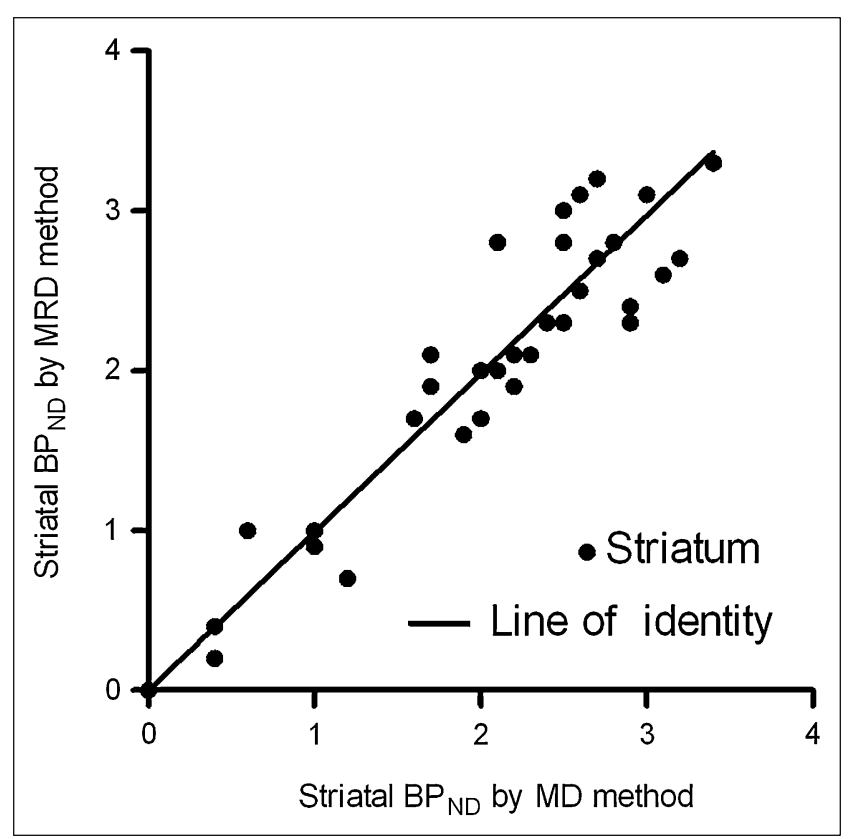

FIGURE 4. $\mathrm{BP}_{\mathrm{ND}}$ values for caudate nucleus and putamen by ROI application with MD vs. probability map-based automatic delineation method. Linear regression analysis showed excellent correlation, $R^{2}=0.96$.

with some uncertainty. Our SBR values are much higher than the $\mathrm{BP}_{\mathrm{ND}}$ values found. This is because $\mathrm{SBR}=\mathrm{BP}_{\mathrm{ND}}+$ 1 , and because our striatal volumes on average are $20 \%$ smaller than the average striatal size of $11.2 \mathrm{~mL}$, because the semiautomatic MRI ROI delineation program does not include the caudate nucleus and putamen as a whole striatum but as 2 separate regions.

The intrasubject variability, depending on the chosen method, was between $11 \%$ and $15 \%$. Data from previous test-retest DAT SPECT studies are reviewed in Table 4; the intrasubject variability and reliability (equivalent to the intraclass correlation coefficient) from the present study fall within the same range.

Not surprisingly, in all studies, except for one, the intrasubject variability was lower in healthy volunteers than in patients with reduced DAT availability.

As previously mentioned, the difference between MD and adjustable-template delineation is limited. Not surprisingly, the similar intraobserver reproducibility of the MD and the SVI methods, compared with the other 2 methods, did not result in a significantly smaller intrasubject variability, even though this method has higher absolute quantification values. A serious limitation with the SVI method as it is currently implemented is that it does not allow for the calculation of a posterior-anterior ratio.

The intrasubject variability of the MRI-defined ROI delineation-the MRD method-yielded an outcome similar to that of the MD method, despite the fact that MRD is most likely better able to determine the exact VOI (17), which could be of particular importance in patients with reduced DAT availability. The volumes with the MRD method were, however, highly reproducible, suggesting that it is because SPECT/MRI coregistration represents a vulnerable step that MRI-template-based delineation lacks a clear benefit (30). Although this coregistration should be similar in patients and in healthy volunteers when using external fiducials, this is apparently not the case. A small misalignment probably has a greater effect on the mean count in a ROI in patients than in healthy volunteers because of the lower striatal radiotracer concentration that can easily move the ROI out of the hot spot. The intrasubject variability of the MD method was almost twice as high in patients as in healthy controls. This is not surprising because it is more difficult to delineate a missing putamen on a SPECT image that is without anatomic information.

TABLE 2

Individual Striatal Intrasubject Variability of Most Affected Side

\begin{tabular}{|c|c|c|c|c|c|c|c|c|c|}
\hline \multirow[b]{2}{*}{ Method } & \multicolumn{8}{|c|}{ Subject } & \multirow[b]{2}{*}{ Mean $\pm \mathrm{SD}$} \\
\hline & 1 & 2 & 3 & 4 & 5 & 6 & 7 & 8 & \\
\hline \multicolumn{10}{|l|}{ MD } \\
\hline Striatal $\mathrm{BP}_{\mathrm{ND}}$ test & 2.06 & 0.87 & 2.07 & 3.21 & 2.11 & 0.57 & 2.58 & 1.64 & 1.9 \\
\hline Striatal $\mathrm{BP}_{\mathrm{ND}}$ retest & 1.88 & 0.87 & 2.55 & 2.66 & 2.63 & 0.58 & 2.56 & 2.05 & 2.0 \\
\hline Reproducibility (\%) & 9.3 & 0.1 & 20.5 & 18.8 & 21.6 & 1.8 & 0.5 & 22.2 & $11.9 \pm 10.0$ \\
\hline \multicolumn{10}{|l|}{ MRD } \\
\hline Striatal $^{\star} \mathrm{BP}_{\mathrm{ND}}$ test & 1.90 & 0.67 & 2.46 & 2.64 & 2.99 & 0.65 & 2.46 & 1.47 & 1.9 \\
\hline Striatal $^{*} \mathrm{BP}_{\mathrm{ND}}$ retest & 1.67 & 0.53 & 2.63 & 2.65 & 2.96 & 0.62 & 3.11 & 2.32 & 2.1 \\
\hline Reproducibility (\%) & 12.9 & 23.3 & 6.6 & 0.7 & 1.1 & 4.1 & 23.3 & 45.0 & $14.6 \pm 15.3$ \\
\hline \multicolumn{10}{|l|}{ SVI } \\
\hline Striatal SBR test & 7.51 & 2.21 & 8.46 & 10.33 & 8.23 & 4.13 & 8.53 & 5.08 & 6.8 \\
\hline Striatal SBR retest & 7.08 & 2.54 & 8.15 & 9.85 & 9.09 & 4.03 & 9.78 & 7.01 & 7.2 \\
\hline Reproducibility (\%) & 5.8 & 14.0 & 3.7 & 4.8 & 9.9 & 2.5 & 13.7 & 32.0 & $10.8 \pm 10.2$ \\
\hline
\end{tabular}

${ }^{*}$ Calculated striatum $=$ volume-weighted (caudate nucleus + putamen).


significant different using MD vs. MRD method ( $t$ test, $P>0.5 ; n=8$ ). 
TABLE 3

Summed Intrasubject Variability from 3 Different ROI Application Methods $(n=8)$

\begin{tabular}{|c|c|c|c|c|}
\hline Method & Caudate nucleus & Putamen & Striatum & ICC \\
\hline $\mathrm{MD}\left(\mathrm{BP}_{\mathrm{ND}}\right)$ & $19.4 \% \pm 14.3 \%$ & $14.8 \% \pm 6.0 \%$ & $11.9 \% \pm 10.0 \%$ & 0.88 \\
\hline $\operatorname{MRD}\left(\mathrm{BP}_{\mathrm{ND}}\right)$ & $16.4 \% \pm 11.9 \%$ & $15.8 \% \pm 11.7 \%$ & $14.6 \% \pm 15.3 \% *$ & 0.90 \\
\hline SVI (SBR) & & & $10.8 \% \pm 10.2 \%$ & 0.90 \\
\hline
\end{tabular}

${ }^{*}$ Calculated striatum $=$ volume-weighted (caudate nucleus + putamen).

ICC $=$ intraclass correlation coefficient.

Unfortunately, we have no data on the SVI method, but the strength of this method is exactly that it requires no anatomic information and, as such, should be independent of the DAT availability.

There was an excellent correlation between $\mathrm{BP}_{\mathrm{ND}}$ values obtained from MD and MRD (Fig. 4). So even though MD of a low-count putamen on the SPECT images is difficult without additional anatomic information, MRI coregistration offers no significant advantage in accuracy when it comes to DAT quantification with SPECT.

Finally, we also compared the 3 ROI delineation methods in terms of variability for the single individual. We found a highly statistically significant correlation between the individual MRD- and SVI-determined intrasubject variability but not with these 2 methods and the MD method. This suggests that compared with the MD, the MRD and the SVI methods are less observer-dependent by either increasing the reproducibility of the ROI volumes (MRD) or simply by bypassing the reproducibility (SVI). With the reduced delineator variation and the strict correlation in the individual patient test-retest variability in these 2 methods, a substantial part of the scan-to-scan variability must be due to changes in DAT availability in the current 2- to 3-wk scanning interval and not to methodologic issues.
A limitation of this study was that the sample size was small; however, it was within the general size of data in test-retest studies for SPECT/PET.

\section{CONCLUSION}

We find that in patients with reduced striatal DAT binding, $\mathrm{MD}$ by an experienced reader provides $\mathrm{BP}_{\mathrm{ND}}$ measurements that are in complete correspondence to MRIbased delineations. Further, the MD and the MRD methods performed equally well in terms of intraobserver reproducibility, despite the fact that the reproducibility of the ROI volumes was superior in the MRI-based method. Thus, the advantage of the ROI volume reproducibility is lost in the coregistration between MR and DAT images. Trying to overcome accurate striatal ROI delineations using templatebased oversized ROIs was not more effective than using $\mathrm{MD}$ in terms of intraobserver reproducibility or intrasubject variability.

\section{ACKNOWLEDGMENTS}

We thank Glenna Skouboe for expert technical assistance. This work was supported by Rigshospitalet; the Lundbeck Foundation; the EC-FP6-project DiMI, LSHB-CT-2005-

TABLE 4

Intrasubject Variability of Various DAT Radioligands for SPECT

\begin{tabular}{|c|c|c|c|c|c|c|}
\hline \multirow[b]{2}{*}{ Study } & \multirow[b]{2}{*}{ Ligand } & \multirow[b]{2}{*}{ Total patients $(n)$} & \multirow[b]{2}{*}{ Delineation } & \multicolumn{2}{|c|}{$\begin{array}{l}\text { Intrasubject } \\
\text { variability* }^{*}\end{array}$} & \multirow[b]{2}{*}{ Reliability ICC } \\
\hline & & & & $\begin{array}{l}\text { Healthy } \\
\text { volunteers }\end{array}$ & Patients & \\
\hline Ziebell et al. (17) & PE2I & 7 & $\mathrm{MD} \mathrm{HC}$ & $4.1 \pm 3.2$ & & 0.96 \\
\hline Seibyl et al. (20) & $\beta-\mathrm{CIT}$ & 7 & Template WS & $12.8 \pm 8.9^{\dagger}$ & & 0.82 \\
\hline Booij et al. (18) & FP-CIT & 6 & Template HC & $7.3 \pm 3.2$ & & $0.92^{\dagger}$ \\
\hline Tsuchida et al. (21) & FP-CIT & 10 & Template HC & $11.1 \pm 10.4$ & & 0.59 \\
\hline Pirker et al. (31) & $\beta-\mathrm{CIT}$ & 9 & $\mathrm{MD} \mathrm{HC}$ & $8.2 \pm 7.2$ & & 0.70 \\
\hline Ziebell et al., current study & PE2I & 8 & MD HC & & $11.9 \pm 10.0$ & 0.88 \\
\hline Seibyl et al. (20) & $\beta-\mathrm{CIT}$ & 7 & Template WS & & $16.8 \pm 13.3^{\dagger}$ & 0.82 \\
\hline Booij et al. (18) & FP-CIT & 6 & Template HC & & $7.9 \pm 6.9$ & $0.72^{\dagger}$ \\
\hline Tsuchida et al. (21) & FP-CIT & 6 & Template HC & & $7.8 \pm 8.9$ & 0.95 \\
\hline Hwang et al. (19) & Trodat & 20 & $\mathrm{MD} \mathrm{HC}$ & & $10.2 \pm 6.2$ & 0.95 \\
\hline${ }^{*}$ Mean outcome for either $\mathrm{BP}_{\mathrm{ND}}$ or SBR $( \pm \mathrm{SD})$ & \multicolumn{6}{|c|}{$\begin{array}{l}{ }^{\dagger} \text { Data extracted from publication. } \\
\text { ICC = intraclass correlation coefficient; } \mathrm{HC}=\text { high-count slides; WS = whole striatum. }\end{array}$} \\
\hline
\end{tabular}


512146; the Toyota Foundation; the University of Copenhagen; and H:S (The Capital Region of Denmark).

\section{REFERENCES}

1. Bao SY, Wu JC, Luo WF, Fang P, Liu ZL, Tang J. Imaging of dopamine transporters with technetium-99m TRODAT-1 and single photon emission computed tomography. J Neuroimaging. 2000;10:200-203.

2. Brucke T, Asenbaum S, Pirker W, et al. Measurement of the dopaminergic degeneration in Parkinson's disease with $\left[{ }^{123} \mathrm{I}\right] \beta$-CIT and SPECT: correlation with clinical findings and comparison with multiple system atrophy and progressive supranuclear palsy. J Neural Transm Suppl. 1997;50:9-24.

3. Prunier C, Bezard E, Montharu J, et al. Presymptomatic diagnosis of experimental parkinsonism with ${ }^{123}$ I-PE2I SPECT. Neuroimage. 2003;19:810816.

4. Emond P, Guilloteau D, Chalon S. PE2I: a radiopharmaceutical for in vivo exploration of the dopamine transporter. CNS Neurosci Ther. 2008;14:47-64.

5. Marshall V, Grosset D. Role of dopamine transporter imaging in routine clinical practice. Mov Disord. 2003;18:1415-1423.

6. Scherfler C, Schwarz J, Antonini A, et al. Role of DAT-SPECT in the diagnostic work up of parkinsonism. Mov Disord. 2007;22:1229-1238.

7. Morrish PK. How valid is dopamine transporter imaging as a surrogate marker in research trials in Parkinson's disease? Mov Disord. 2003;18(suppl 7):S63S70.

8. Poewe W, Scherfler C. Role of dopamine transporter imaging in investigation of parkinsonian syndromes in routine clinical practice. Mov Disord. 2003;18(suppl 7):S16-S21.

9. Knudsen GM, Karlsborg M, Thomsen G, et al. Imaging of dopamine transporters and $\mathrm{D}_{2}$ receptors in patients with Parkinson's disease and multiple system atrophy. Eur J Nucl Med Mol Imaging. 2004;31:1631-1638.

10. Innis RB, Seibyl JP, Scanley BE, et al. Single photon emission computed tomographic imaging demonstrates loss of striatal dopamine transporters in Parkinson disease. Proc Natl Acad Sci USA. 1993;90:11965-11969.

11. Marek K, Jennings D, Seibyl J. Do dopamine agonists or levodopa modify Parkinson's disease progression? Eur J Neurol. 2002;9(suppl 3):15-22.

12. Zaidi H, El Fakhri G. Is absolute quantification of dopaminergic neurotransmission studies with ${ }^{123}$ I SPECT ready for clinical use? Eur J Nucl Med Mol Imaging. 2008;35:1330-1333.

13. Delorenzo C, Kumar JD, Zanderigo F, Mann JJ, Parsey RV. Modeling considerations for in vivo quantification of the dopamine transporter using $\left[{ }^{11} \mathrm{C}\right]$ PE2I and positron emission tomography. J Cereb Blood Flow Metab. 2009;29:1332-1345.

14. Koch W, Radau PE, Hamann C, Tatsch K. Clinical testing of an optimized software solution for an automated, observer-independent evaluation of dopamine transporter SPECT studies. J Nucl Med. 2005;46:1109-1118.

15. Tossici-Bolt L, Hoffmann SM, Kemp PM, Mehta RL, Fleming JS. Quantification of $\left[{ }^{123}\right.$ I]FP-CIT SPECT brain images: an accurate technique for measurement of the specific binding ratio. Eur J Nucl Med Mol Imaging. 2006;33:1491-1499.

16. Seibyl JP, Laruelle M, van Dyck CH, et al. Reproducibility of iodine-123- $\beta$-CIT SPECT brain measurement of dopamine transporters. J Nucl Med. 1996;37:222228.

17. Ziebell M, Thomsen G, Knudsen GM, et al. Reproducibility of $\left[{ }^{123} \mathrm{I}\right] \mathrm{PE} 2 \mathrm{I}$ binding to dopamine transporters with SPECT. Eur J Nucl Med Mol Imaging. 2007;34:101-109.

18. Booij J, Habraken JB, Bergmans P, et al. Imaging of dopamine transporters with iodine-123-FP-CIT SPECT in healthy controls and patients with Parkinson's disease. J Nucl Med. 1998;39:1879-1884.

19. Hwang WJ, Yao WJ, Wey SP, Ting G. Reproducibility of ${ }^{99 m}$ Tc-TRODAT-1 SPECT measurement of dopamine transporters in Parkinson's disease. $\mathrm{J} \mathrm{Nucl}$ Med. 2004;45:207-213.

20. Seibyl JP, Marek K, Sheff K, et al. Test/retest reproducibility of iodine-123- $\beta$ CIT SPECT brain measurement of dopamine transporters in Parkinson's patients. $J$ Nucl Med. 1997;38:1453-1459.

21. Tsuchida T, Ballinger JR, Vines D, et al. Reproducibility of dopamine transporter density measured with ${ }^{123}$ I-FPCIT SPECT in normal control and Parkinson's disease patients. Ann Nucl Med. 2004;18:609-616.

22. Pinborg LH, Ziebell M, Frokjaer VG, et al. Quantification of ${ }^{123}$ I-PE2I binding to dopamine transporter with SPECT after bolus and bolus/infusion. J Nucl Med. 2005;46:1119-1127.

23. Small AD, Prosser J, Motherwell DW, McCurrach GM, Fletcher AM, Martin W. Downscatter correction and choice of collimator in ${ }^{123} \mathrm{I}$ imaging. Phys Med Biol. 2006;51:N307-N311.

24. de Nijs R, Svarer C, Holm S. Correction for high energy photons and scatter for dynamic SPECT studies with I-123 [abstract]. Eur J Nucl Med Mol Imaging. 2004;31(suppl 2):402.

25. Kretschmann H-J, Weinrich W. Neuroanatomy and Cranial Computed Tomography. Stuttgart, Germany: Thieme; 1986.

26. Svarer C, Madsen K, Hasselbalch SG, et al. MR-based automatic delineation of volumes of interest in human brain PET images using probability maps. Neuroimage. 2005;24:969-979.

27. Innis RB, Cunningham VJ, Delforge J, et al. Consensus nomenclature for in vivo imaging of reversibly binding radioligands. J Cereb Blood Flow Metab. 2007;27:1533-1539.

28. Ichise M, Ballinger JR, Vines D, Tsai S, Kung HF. Simplified quantification and reproducibility studies of dopamine D2-receptor binding with iodine-123-IBF SPECT in healthy subjects. J Nucl Med. 1997;38:31-37.

29. van Dyck CH, Seibyl JP, Malison RT, et al. Age-related decline in dopamine transporters: analysis of striatal subregions, nonlinear effects, and hemispheric asymmetries. Am J Geriatr Psychiatry. 2002;10:36-43.

30. Marner L, Knudsen GM, Haugbol S, Holm S, Baare W, Hasselbalch SG. Longitudinal assessment of cerebral $5-\mathrm{HT}_{2 \mathrm{~A}}$ receptors in healthy elderly volunteers: an $\left[{ }^{18} \mathrm{~F}\right]$-altanserin PET study. Eur J Nucl Med Mol Imaging. 2008;35:1-4.

31. Pirker W, Djamshidian S, Asenbaum S, et al. Progression of dopaminergic degeneration in Parkinson's disease and atypical parkinsonism: a longitudinal $\beta$-CIT SPECT study. Mov Disord. 2002;17:45-53. 